duration less than 24 hours and recovery is complete. The author emphasizes that the clinical features of hypoxic-ischemic encephalopathy are not specific and that similar symptoms may be caused by metabolic disorders, infection or cerebral dysgenesis.

\title{
BIRTH ASPHYXIA, CEREBRAL METABOLISM AND HEAD SIZE
}

Studies of cerebral oxidative metabolism were carried out by phosphorus magnetic resonance spectroscopy during the first week of life in 52 infants with birth asphyxia admitted to the Neonatal Unit at University College Hospital, London. Cerebral phosphocreatine/inorganic phosphate concentration ratio was used as an index of oxidative metabolism and correlated with neurodevelopmental outcome and head growth at 1 year. (Roth SC et al. Relation between cerebral oxidative metabolism following birth asphyxia, and neurodevelopmental outcome and brain growth at one year. Dev Med Child Neurol April 1992; 34:285-295.) (Correspondence: Dr. Simon C. Roth, Department of Paediatrics, University College and Middlesex School of Medicine, The Rayne Institute, 5 University Street, London WC1E 6JJ, England.)

COMMENT. The use of these neuroimaging and biochemical techniques should help in the prediction of outcome following neonatal encephalopathy. However, the complexity of the techniques may detract from their value in practice. (Bax M. Editorial. Birth asphyxia. Dev Med Child Neurol 1992; $\underline{34}$ :283-284.)

Central diabetes insipidus as an unusual complication of hypoxic brain damage is described in 2 children at the Department of Pediatrics, Juntendo University School of Medicine, Tokyo, Japan (Arisaka O et al. Child's Nerv Syst March $1992 ; \underline{8}: 81-82)$. Both patients developed cardiopulmonary arrest after choking and both had hypernatremia and low urinary antidiuretic hormone concentrations in the terminal stages. The most common causes of central diabetes insipidus are tumors or trauma in the neurohypophyseal area.

\section{NEUROMUSCULAR DISEASES}

\section{MYOTONIC DYSTROPHY: SEVERITY AND MATERNAL AGE}

The severity of myotonic dystrophy in 17 affected sibling pairs from 15 families in which 2 or more affected children were born to mothers with myotonic dystrophy is reported from the Hospital for Sick Children, London and the Prince of Wales Children's Hospital, Sydney, Australia. In 13 of 17 sibling pairs the younger child was more severely affected than the older child. Increasing age difference between the affected siblings paralleled increasing age for each mother and showed a positive correlation with the difference in disease severity between siblings. Infants born to older mothers suffered more severe myotonic dystrophy. Maternal age at delivery correlated with the age at which the infant sat alone and walked alone. In addition, the incidence of neonatal feeding difficulties, neonatal respiratory difficulties, surgery for talipes, and scoliosis were directly related to maternal age at delivery. (Andrews, PI, Wilson, J. Relative disease severity in siblings with 
myotonic dystrophy. J Child Neurol April 1992; $\underline{7}: 161-167$.$) (Correspondence:$ Dr. P. Ian Andrews, Box 3533, Duke University Medical Center, Durham, NC 27710.)

COMMENT. These findings have relevance in genetic counseling and suggest that maternal age and birth order are important in the relative severity of childhood onset myotonic dystrophy. The authors note that a normal infant born after a neonatally affected child is unlikely to develop myotonic dystrophy.

An increased risk of spontaneous abortion, cesarian section and neonatal death for women with myotonic dystrophy is reported from the Chicoutimi Hospital, Quebec Canada (Prevost C, Mathieu J. Pregnancy outcome of women with myotonic dystrophy. Neurology April 1992; 42 Suppl $3: 226)$. The risk estimate that women with myotonic dystrophy will have a congenitally effected child is $3-9 \%$. Among children surviving the neonatal period, $7.3 \%$ had congenital myotonic dystrophy and $7.3 \%$ had childhood onset myotonic dystrophy.

\section{DYSTROPHINOPATHY IN FEMALES}

Dystrophin immunocytochemistry was used to study muscle biopsies from 505 girls and women with neuromuscular disease seen at the National Institute of Neuroscience, Tokyo, the University of Pittsburgh School of Medicine and the Columbia Presbyterian Medical Center, New York. The laboratory findings of $25(54 \%)$ of 46 isolated cases with muscle biopsies were consistent with the familial cases and all showed myopathic histopathology and abnormal elevations of serum CK. The clinical presentations of the isolated cases were consistent with familial cases and included proximal limb weakness before age $10(40 \%)$, myalgias or cramps $(24 \%)$ and grossly elevated CK levels (24\%). About $10 \%$ of the isolated cases of hyperCKemic myopathy were proven by dystrophin analysis to have a dystrophinopathy as the cause of their disease (manifesting carriers of Duchenne dystrophy). (Hoffman EP et al. Dystrophinopathy in isolated cases of myopathy in females. Neurology May $1992 ; \underline{42}: 967-975$.) (Reprints: Dr. E.P. Hoffman, University of Pittsburgh School of Medicine, Biomedical Science Tower, Pittsburgh, PA 15261; Dr. K. Arahata, National Institute of Neuroscience, 4-1-1 Ogawahigahi-cho, Kodaira, Tokyo 187, Japan; or Dr. E. Bonilla, Department of Neurology, ColumbiaPresbyterian Medical Center, New York, NY 10032.)

COMMENT. All isolated female dystrophinopathy patients had had other diagnoses prior to dystrophin testing, the most common being the limb girdle muscular dystrophy. The clinical criteria that implicate a dystrophinopathy in isolated nonfamilial cases in girls and women include a serum CK level in excess of 1,000 IU/l and a myopathic histopathology. The exclusion of dystrophinopathy by dystrophin analysis is necessary in confirming a diagnosis of isolated limb girdle muscular dystrophy.

A dystrophin test is also recommended in patients with idiopathic cardiomyopathies since cardiomyopathy was found as the only clinical manifestation in 2 Duchenne muscular dystrophy carriers reported from 